CWRU-P15-96

October 1996

\title{
DIRTY BLACK HOLES AND HAIRY BLACK HOLES
}

\author{
Lawrence M. Krauss 円, Hong Liu \\ Department of Physics \\ Case Western Reserve University \\ 10900 Euclid Ave., Cleveland, OH 44106-7079 \\ and \\ Junseong Heo \\ Physics Dept. \\ Yale University \\ New Haven CT 06511
}

\begin{abstract}
An approach based on considerations of the non-classical energy momentum tensor outside the event horizon of a black hole provides additional physical insight into the nature of discrete quantum hair on black holes and its effect on black hole temperature. Our analysis both extends previous work based on the Euclidean action techniques, and corrects an omission in that work. We also raise several issues related to the effects of instantons on black hole thermodynamics and the relation between these effects and results in two dimensional quantum field theory.
\end{abstract}

\footnotetext{
${ }^{1}$ also Department of Astronomy
} 


\section{Introduction}

Semiclassical considerations of quantum fields in curved space backgrounds have revolutionized our thinking about both classical and quantum gravity.(i.e. [1, 2]) A great deal of work has been carried out in the past decade aimed at addressing potential problems of untarity violation and information loss. The jury is still out, but during this process many new insights have been gained both about black hole physics, and about possible new Planck Scale phenomena, and phenomena in higher dimensions.

One result which relies only on phenomena which exist well below the Planck scale has been the recognition that black holes can harbor "quantum hair" (i.e. [3, [, 5]) - that is, quantum mechanical observables can exist associated with black holes beyond those allowed by the classical "no hair theorems" [7, 8, 9].

The prototype example of such quantum hair [四] is quite simple. Consider an abelian $\mathrm{U}(1)$ gauge theory containing two matter fields $\eta$ and $\phi$ with charge $N e$ and $e$ respectively. If the field $\eta$ condenses at some energy scale $v$, then the gauge field will become massive by the Higgs mechanism, and below this scale the effective theory involving only the light field $\phi$ will have a residual discrete $Z_{N}$ symmetry. This low energy broken symmetry theory also can contain stable strings threaded by magnetic flux $2 \pi / N e$. The scattering of $\phi$ quanta, with charge $e$ from such strings is dominated by the Aharonov-Bohm effect [10, 11] involving quantum phases uniquely determined by the product of charge and flux, and thus allowing a determination of the total charge modulo $\mathrm{N}$ which scatters off the string.

Since the quantum phases in question are global quantities, a $\phi$ quanta 
which falls into a black hole will be measurable as such even after it falls inside the event horizon of the black hole [4, 12, 13]. Such a charged black hole therefore has "discrete gauge quantum hair". [4, 12, 5]

Given the semiclassical relationship between entropy, area, and temperature for black holes, one might expect that any restriction on the number of microstates associated with a given classical black hole macrostate, as would occur if one could measure additional black hole quantum numbers, would have a concomitant effect on the black hole's entropy, and hence its temperature. In a beautiful series of papers, Coleman, Preskill and Wilczek explored this possibility [14, 5, 6]. They uncovered an (exponentially small) effect on temperature, but surprisingly the sign of the temperature change depended upon the relative scales of the spontaneous symmetry breaking associated with the quantum hair, and the inverse size of the black hole event horizon. Moreover, they also uncovered a new observable associated with quantum hair: a non-classical electric field could exist and be measured outside of the event horizon.

Motivated by the recognition that a non-classical field exists outside the event horizon of a black hole endowed with discrete gauge hair, we focus here on the energy momentum tensor outside the black hole. In this way, one might hope to use Minkowski space arguments to get some additional insight into the physics behind the effects of quantum hair which might not be manifest in a Euclidean Action approach. An appropriate Minkowskispace formalism was developed by Visser [15] for treating "dirty" black holes, where non-zero matter fields exist outside the event horizon. However, since the electric field associated with discrete hair is non-classical, in the sense 
that it is not a solution of the coupled vacuum Einstein-Maxwell equations, standard methods such as Visser's, which require such solutions, cannot be applied directly.

While the electric field generated outside the event horizon is not a solution of the Minkowski field equations, the individual instantons whose contributions sum to produce such a field are solutions of the coupled Euclidean Einstein-Maxwell equations. This suggests a hybrid approach, in which we use the Visser formalism in Euclidean space, and then focus on the effect of individual instantons, recognizing of course that their contribution is negligible except to quantities which explicitly depend on the discrete gauge charge, and which vanish in perturbation theory.

The Euclidean spacetime metric generated by a static spherically symmetric distribution of matter can be put in the form:

$$
d s^{2}=e^{-2 \phi(r)}\left(1-\frac{b(r)}{r}\right) d t^{2}+\left(1-\frac{b(r)}{r}\right)^{-1} d r^{2}+r^{2} d \Omega^{2}
$$

With the assumption that the metric has an asymptotically flat geometry and an event horison, boundary conditions can be imposed as: $\phi(\infty)=$ $0, b(\infty)=2 G M_{B H}, b\left(r_{H}\right)=r_{H}$ where $M_{B H}$ is the mass of the black hole and $r_{H}$ is the horizon size. Einstein's equations can then be solved formally to give $b(r)$ and $\phi(r)$ in terms of the components of the energy momentum tensor. Defining

$$
T_{t}^{t}=\rho, \quad T_{r}^{r}=\tau, \quad T_{\theta}^{\theta}=T_{\varphi}^{\varphi}=-\mu .
$$

the Hawking temperature and the horizon size of the black hole can be expressed as (i.e. see [15]):

$$
\frac{1}{\beta \hbar}=\frac{1}{4 \pi r_{H}} e^{-\phi\left(r_{H}\right)}\left(1-b^{\prime}\left(r_{H}\right)\right)
$$




$$
r_{H}=2 G M_{B H}+8 \pi G \int_{r_{H}}^{\infty} d r \rho r^{2}
$$

When the external matter contribution to the geometry is much smaller than that of the black hole, as will be the case of interest here, equations (1) and (2) can be systematically expanded and the lowest order corrections to black hole thermodynamics can then be obtained. Define

$$
A=\frac{8 \pi G}{2} \int_{r_{H}}^{\infty} \frac{\rho-\tau}{r-r_{H}} r^{2} d r ; B=8 \pi G \rho_{H} r_{H}^{2} ; m=4 \pi \int_{r_{H}}^{\infty}(2 \mu+\rho-\tau) r^{2} d r
$$

Expanding to first order in $A, B$, and $\left(m / M_{B H}\right)$ and using energy conservation one can derive an expression for $\beta \hbar$ in terms of only the components of the energy momentum tensor and $M_{B H}$ :

$$
\beta \hbar=8 \pi G M_{B H}\left(1+\frac{m}{M_{B H}}-2(A+B)+\ldots\right)
$$

Examining (3) it is now clear that the sign of the correction to the black hole temperature, for fixed mass, depends upon the relative sign of the term $m / M_{B H}-2(A+B)$. It is precisely this result which establishes the connection between the Weak Energy Condition (WEC), and the effect of fields outside the event horizon to the black hole temperature. All forms of classical matter which satisfy the WEC also satisfy the relation $m / M_{B H}-2(A+B) \geq 0$, implying that classical matter outside the black hole can only lower the temperature 15]. However, as we shall see, instanton contributions need not be of this form. Indeed, the non-classical electric field outside a black hole endowed with discrete hair is precisely a manifestation of the fact that quantum effects can violate the WEC.

We now turn to the Euclidean Einstein-Abelian-Higgs system, the prototypical example of quantum hair. This system has solutions corresponding 
to a vortex sitting in the 2-d Euclidean $r-t$ plane of a black hole. The two other Euclidean dimensions $\theta, \phi$, (which would correspond to $z, t$ for a corresponding vortex in Minkowski space) are suppressed. As emphasized by by CPW, in a Euclidean path integral formalism these instanton solutions play a central role in producing the observable non-classical effects of discrete charge outside of the black hole event horizon, as the sum over these instantons includes Aharonov-Bohm phases which are sensitive to the discrete charge contained the black hole. We wish to examine this effect in the context of the formalism we have described above.

We use standard ansatz for these vortex solutions: $\phi=v f(r) e^{-i \frac{2 \pi}{\beta \hbar} t}, A_{t}=$ $\frac{2 \pi}{\beta \hbar} \frac{1}{e}(1-a(r))$, with boundary conditions: $f\left(r_{H}\right)=0, f(\infty)=1, a\left(r_{H}\right)=$ $1, a(\infty)=0 . \quad A_{t}$ satifies an equation which reflects the flux quantization condition for vortices in the broken phase: $\left.e \int_{0}^{\beta \hbar} d t A_{t}\right|_{r=\infty}=2 \pi$. Following CPW one can consider two limiting cases, depending upon whether the vortex width is much larger or smaller than the size of the event horizon. The virtue of equation 3 is that it lends itself directly to such an analysis. Competition among the different terms as their $r$-dependence varies, can lead, in different limits, to a different sign for the correction to the black hole temperature. What actually occurs, however depends subtlely yet crucially on the nature of the vortex solution in curved space, as we shall demonstrate below.

The thin string limit is particularly simple to analyze in this context. In the thin string limit, the vortex width $r_{s} \ll r_{H}$, so that,

$$
\begin{aligned}
2(A+B)-\frac{m}{M_{B H}} & \approx \frac{8 \pi G}{r_{H}} \int_{r_{H}}^{\infty}\left(4 \mu r r_{H}-2 \mu r^{2}\right) d r \\
& \approx 16 \pi G r_{H} \int_{r_{H}}^{\infty} \mu d r
\end{aligned}
$$

Then the correction to the Hawking temperature due to the vortex instanton 
can directly be expressed as:

$$
\beta \hbar \doteq 8 \pi G M_{B H}\left[1-16 \pi G r_{H} \int_{r_{H}}^{\infty} \mu d r\right]
$$

In this limit, the vortex lies in the region $r \sim r_{H}$. One can then show (see [16] for further details) that $A \sim B \sim \frac{v^{2}}{M_{p l}^{2}}$ and thus the correction to the Hawking temperature:

$$
\beta \hbar \sim 8 \pi G M_{B H}\left[1-O\left(\frac{v^{2}}{M_{p l}^{2}}\right)\right]
$$

We see that the effect of a single instanton in this limit is to raise the black hole temperature. Of course, we emphasize that to determine the thermal effect of discrete charge one must sum over instantons, and thus go beyond our formalism. (Note that if one does the summation[5], the interference between instantons and anti-instantons for the weighted action produces an effect which is opposite in sign to that for the single instanton). Nevertheless, the single instanton contribution to the temperature which we calculate using the energy momentum formalism directly is identical with that determined by CPW in the thin string case based on their estimates of the deficit angle and contributions to the action.

The thick string limit, in which $r_{s}>>r_{H}$, is much more subtle, precisely because in this limit the curvature associated with the sphere at the event horizon cannot be ignored, as in the thin string limit. Put another way, we cannot accurately picture the instanton as a vortex simply living in the two dimensions of a flat $r-t$ plane. If this persisted to be the case, one could use well known properties of vortex solutions inside the core of the vortex, where the symmetry is unbroken, along with the boundary conditions associated with the magnetic flux carried in the core, to examine (3), and estimate the 
instanton contribution. However, if one does this, (as we confess we first did), several anomalies arise. In the first place, the lowest order correction one finds to the black hole temperature is proportional to $v^{2} / M_{p l}^{2}$, while CPW focus on a zeroth order contribution in this limit. In the second place, a straightforward application of this ansatz to a calculation of the action, which we always use as a check of our approximation, yields a result which appears to be nonsensical - namely that the instanton action is less than the Schwarzchild action.

The resolution of this paradox lies in consideration of the effects of curved space, associated with the spherical surface at the event horizon. In this case, the vortex core behaves remarkably differently from the flat space vortex (for further discussion see [16]). The presence of extra $r^{2}$ contributions in the spherical derivatives around the event horizon allow a vortex solution in which both the gauge potential and magnetic field fall off exactly as in the unbroken theory inside the core, so that the physics inside becomes largely insensitive to the boundary conditions associated with the Higgs field behavior at the vortex surface. In other words, as $v \rightarrow 0$ the thick string limit smoothly approaches the Reissner-Nordstrom case-there are no singular effects due to boundary conditions at infinity in the curved space solution.

Without solving in detail for the vortex solution here, the net effect of the analysis is that no spatial derivatives in solutions blow up at infinity, so that if we define the quantities

$$
y=\frac{r}{r_{H}}, \quad \epsilon^{2}=2 e^{2} r_{H}^{2} v^{2}, \quad \beta_{0}=\frac{\lambda}{2 e^{2}}
$$

and explicity define the thick string limit by letting $\epsilon^{2}$ tend to zero, as the vortex becomes thicker and thicker with $\epsilon^{2}$ smaller and smaller, the action 
reduces to that of Euclidean Reissner-Nordstrom black hole, and there are no small or large parameters other than $\epsilon^{2}$ in the energy momentum tensor. We can thus expand these quantities in terms of $\epsilon^{2}: \rho=\rho_{0}+\epsilon^{2} \rho_{1}+\cdots, \tau=$ $\tau_{0}+\epsilon^{2} \tau_{1}+\cdots, \mu=\mu_{0}+\epsilon^{2} \mu_{1}+\cdots$

One may wonder why one should bother to consider corrections of order $\epsilon^{2}$ if the zeroth order term in $\epsilon^{2}$ (the one which is calculated by CPW) indeed gives the dominant contribution. The point is that this does not seem to be guaranteed to be the case

The contributions from the zeroth order terms are identical to those in the case of the Euclidean Reissner-Nordstrom black hole [15, 5] so that

$$
\beta \hbar \approx \sim 8 \pi G M_{B H}\left(1+O\left(\frac{M_{p l}^{4}}{M_{B H}^{4}}\right)+\cdots\right)
$$

The contributions from the first order terms yield:

$$
\beta \hbar=8 \pi G M_{B H}\left(1-\frac{G}{4 e^{2} r_{H}^{2}} \epsilon^{2} T+\cdots\right)
$$

where $\mathrm{T}$ is a dimensionless quantity of $O(1)$ derived from (3) as

$$
T=\frac{4 e^{2} r_{H}}{8 \pi} \int_{r_{H}}^{\infty} d r\left[\left(4 \mu_{1} r r_{H}-2 \mu_{1} r^{2}\right)-\left(\rho_{1}-\tau_{1}\right) r\left(r-r_{H}\right)\right]
$$

Now we can compare the results from the zeroth order terms and the first order terms. The ratio of the first order (in $\epsilon^{2}$ ) contribution correction to the zeroth order (to both the Hawking Temperature and the action) is

$$
\gamma=\frac{\epsilon^{2} M_{B H}^{2}}{M_{p l}^{2}}=v^{2} M_{B H}^{4} / M_{p l}^{6}
$$

Now, recall that the thick string limit is

$$
\epsilon^{2}=v^{2} M_{B H}^{2} / M_{p l}^{4} \ll 1 .
$$


However, for the semiclassical analysis of black hole thermodynamics to be meaningful, $M_{p l}^{2} / M_{B H}^{2}$ has to be very small. For sufficiently massive black holes, it is certainly possible that both $\epsilon^{2} \ll 1$ and $\gamma \gg 1$, in which case the contributions from the the first order terms cannot be neglected. For example, if we keep $\epsilon^{2}$ fixed but let $\left(M_{B H} / M_{p l}\right)^{2} \rightarrow \infty$ (which requires also making $v^{2} \rightarrow 0$ ), then $\gamma \rightarrow \infty$, so that while both the zeroth and first order contributions go to zero, the first order piece becomes arbitrarily large compared to the second. Stated another way, the limit in which only the Reissner-Nordstrom piece is considered, as was done by CPW, is not the generic thick string limit, but is rather the limit $\gamma \ll 1$.

It is perhaps surprising that for sufficiently large black holes the first order terms in $\epsilon^{2}$ may become comparable or larger than the zeroth order terms. However recalling our heuristic discussion earlier in this section, this effect is perhaps understandable. For larger black holes the curvature at the event horizon becomes progressively smaller. While it may be true that the $v \rightarrow 0$ limit goes smoothly to the Reissner-Nordstrom case, increasing the black hole mass reduces the curvature effects at the horizon which are responsible for the domination of the Reissner-Nordstrom contribution compared to vortex symmetry breaking contribution proportional to the vev of the Higgs fieldnamely, $\epsilon^{2}$ must be correspondingly reduced as the black hole mass increases in order for the first order contribution in $\epsilon^{2}$ to be negligible.

Finally then, we may ask what the sign of the first order term in the expression for the black hole temperature given above is. The sign of $T$ is in general indeterminate. However, if we make the anzatz that the first order term takes a form similar to that which would occur for the flat space 
vortex, one can show that this quantitity is manifestly negative, and hence the contribution of this piece to the Hawking temperature would be of the same sign as the zeroth order contribution--namely instantons in the thick string limit generically cool down a black hole, the opposite of the thin string result. Thus the general observation of CPW on the nature of the effect is correct, even if the overall order of the dominant contribution may not be what they calculated. Note, also that in all cases the dominant contribution to the action is positive, so the semiclassical instanton approximation is stable.

Our results indicate that one may fruitfully extend Minkowski space methods designed to probe the effects of classical fields outside the event horizon on the thermodynamical properties of black holes to the Euclidean regime of semiclassical phenomena. This allows a more intuitive physical picture of the origin of such effects. It may be useful in exploring the nature of other semiclassical contributions to black hole thermodynamics beyond those considered here associated with quantum hair.

We conclude with a remark which is more relevant to the Euclidean formalism directly, and to the instanton sum which results in a non-classical electric field outside the event horizon. Such a non-classical electric field induced by instanton effects is not unique to black holes. Indeed, the prototypical example occurs in a two dimensional Abelian Higgs model. In this case, in the presence of a topological term, Euclidean instantons induce a non-zero non-classical background electric field (i.e. [17]). Note that there are no corresponding Euclidean instantons in the four dimensional Abelian Higgs model in flat space. However, the presence of a black hole event horizon alters the topology of the corresponding Euclidean continuation so that 
instantons of the type examined here both exist, and, if the black hole is charged, can produce observable effects. We believe the analogy between the two dimensional Abelian Higgs model with a topological term and a black hole with discrete hair can be made exact, and are currently exploring this issue [16]. If this is the case, discrete hair may be cast in a different light, which may bear a closer relation to other results associated with two dimensional field theories.

We would like to thank Sidney Coleman, John Preskill, Tanmay Vachaspati and Frank Wilczek for discussions and essential insights. LMK and HL are supported by the DOE and funds from Case Western Reserve University.

\section{References}

[1] S. W. Hawking, Phys. Rev. D14, 2460 (1976)

[2] J. Preskill, Phys. Scrip. T36, 258 (1991)

[3] M. J. Bowick et al Phys. Rev. Lett. 61, 2823 (1988)

[4] L. M. Krauss and F. Wilczek, Phys. Rev. Lett. 62, 1221 (1989)

[5] S. Coleman, J. Preskill, and F. Wilczek, Nucl. Phys. B387, 175 (1992)

[6] S. Coleman, J. Preskill, and F. Wilczek, Mod. Phys. Lett. A6, 1631 (1991), Phys. Rev. Lett. 67, 1975 (1991)

[7] J. D. Bekenstein, Phys. Rev. D5, 1239, 2403 (1972) 
[8] C. Teitelboim, Phys. Rev. D5, 2941 (1972)

[9] S. L. Adler and R. B. Pearson, Phys. Rev. D18, 2789 (1978)

[10] Y. Aharonov and D. Bohm, Phys. Rev. 119, 485 (1959)

[11] M. G. Alford and F. Wilczek, Phys. Rev. Lett. 62, 1071 (1989)

[12] J. Preskill and L.M. Krauss, Nucl. Phys. B341, 50 (1990)

[13] M.G. Alford, J. March-Russell and F. Wilczek, Nucl. Phys. B337, 695 (1990)

[14] S. Coleman, L. M. Krauss, J. Preskill, and F. Wilczek, J. Gen. Rel. Grav., (1991)

[15] M. Visser, Phys. Rev. D46, 2445 (1992)

[16] L.M. Krauss, H. Liu, in preparation.

[17] R. Rajaraman, Solitons and Instantons (North-Holland, 1982); S. Coleman, The use of instantons, in Aspects of Symmetry (Cambridge, London, 1985) 